\title{
The Role of Teriparatide in the Management of Fracture Non-Union: A Systematic Review of Case Reports
}

\author{
$\$$ \\ Ervin Chino N. Tayag, MD, \\ Charles Abraham C. Villamin, MD
}

\begin{abstract}
Teriparatide has been known to aid in the treatment of osteoporosis but its use in the management of fracture disorders is poorly documented. This review aims to show that teriparatide administration may improve the healing process in fractures that fail to unite after sustaining trauma. A total of 22 reported cases have been identified from 2009 to 2017. Teriparatide doses were given in a median duration of 5.6 months with a median time to complete union of 8.5 months. This review systematically summarizes all clinical case reports of non-union treated with teriparatide for us to gain insight into its off-label use.
\end{abstract}

Keywords: Nonunion, teriparatide, PTH

\section{INTRODUCTION}

Fractures are a common occurrence and have a significant impact on the quality of life for patients and considerable economic consequences for our society. It is estimated that 7.9 million fractures occur annually in the United States. Healing occurs via rapid bone regeneration followed by osteoclast-mediated remodeling resulting in new bone with structural integrity and a geometrical configuration similar to that prior to the injury. In the majority of cases, healing

Ervin Chino Tayag

tsino.town@gmail.com

University of Santo Tomas Hospital, Manila, Philippines is uneventful, but in $5-10 \%$ of cases delayed or impaired healing occurs. [3]

Non-union of a fracture represents the most dramatic example of poor healing as complete restoration fails in the absence of some form of treatment. Thus, the normal biological healing process is deficient in achieving complete repair.

There are several conditions affecting the rate of fracture healing: they are divided into general and local factors. In the first group there are age, type of bone involved, nutritional status, drug therapy and bone pathology: speed of fracture union decreases with skeletal maturity and also if the patient is not in good nutritional status, many drugs and pre-existing bone pathology adversely affect fracture healing such as corticosteroids, nonsteroidal anti-inflammatory drugs (NSAID) or osteogenesis imperfecta and others metabolic disease of bone. Local factors are the separation of bone ends, the disturbance of blood supply, infections, the property of bone involved and type of fracture: bone ends must not be separated or with soft tissue interposition, blood supply is fundamental and its interruption may lead to necrosis, pathogens interact with reparative cellular activity resulting in a prolonged inflammatory phase and finally, any type and localization of fracture heals according to their intrinsic characteristics.[2]

Movement of the fracture site interferes with vascularization of hematoma, disrupts bridging callus and endosteal bone growth. On the other side, an optimal balance between stability and micro-motion encourages callus formation and produces electrical 
local currents which improve bone healing by stimulating an inflammatory-type response and promoting calcification of fibrocartilage. Low-intensity pulsed ultrasound plays a relevant role in the biomechanical environment; it accelerates fracture healing perhaps by acting directly on proliferation and differentiation of multipotent human bone marrow derived mesenchymal stromal cells. Actually, the use of platelet-rich plasma (PRP) seems to play an important role in fracture healing and favors fusion processes in spinal arthrodesis. Due to their osteoconduction activity, bone substitutes too such as hydroxyapatite and tricalcium phosphate represent an important resource to be used in delayed bone healing and atrophic non-union with a large bone gap.[2]

The definition of non-union in fracture healing is a disturbance of the normal repairing process with the expectation that no consolidation will be achieved without focused and accurate treatment. Non-union is defined according to the American Food and Drug Administration (FDA 1988) as "established when a minimum of nine months has elapsed since injury and the fracture shows no visible progressive signs of healing for three months". This definition is not always true because the healing time of a fracture depends on the bone concerned, for example, a diaphyseal fracture of the femur needs at least four to six months to heal. In some cases, it needs more time, especially if the fracture is open or comminuted. On the other hand, a femoral neck fracture can be considered as a non-union after only three months.[2]

Teriparatide (TPTD), a human parathyroid hormone (PTH) analog, is currently the only available anabolic agent. PTH plays a major role in orchestrating bone remodeling by modulating the bone marrow microenvironment and regulating osteogenic signaling pathways. TPTD has been shown to increase bone mass, improve bone quality and reduce the risk of fracture in patients with severe osteoporosis. Several reports have also demonstrated its efficacy in the treatment of fracture nonunion. [17]

In 2009, Bukata, et al. published a report of anecdotal clinical experience wherein they had 145 patients, the majority of whom (88\%) had failed previous fracture healing treatment, presented with their current fracture as a non-union or delayed union, or were elderly and high-risk candidates for surgical intervention for their fracture sites. Fifty percent of the patients had persistent pain and fracture healing delay for at least six months before starting TPTD.
Within 12 weeks of starting TPTD, 97\% (141/145) of patients experienced pain resolution at their fracture or fusion site and $93 \%(135 / 145)$ of patients had radiographic and clinical union of their fractures. TPTD treatment was well-tolerated. These data should be considered in light of the limitations of single-site anecdotal experience.

In this review, we want to focus on some of the aspects shown in non-union that seem to be potential biomechanical and biochemical environment targets for TPTD.

Due to the lack or absence of systematic reviews or meta-analyses on the role of TPTD in the management of fracture nonunion, the author aimed to investigate the effect of TPTD therapy in the setting of a fracture nonunion by reviewing the current evidence on the effectiveness of TPTD in the management of fracture nonunions.

\section{CRITERIA FOR CONSIDERING STUDIES FOR THIS REVIEW}

\section{Types of studies}

To determine the role of TPTD in fracture nonunions, case reports were considered in the absence of randomized clinical trials (RCT).

\section{Type of participants}

This review included case reports with adults, both male and female, from 25 to 88 years of age who had fractures of either the femur, tibia or vertebra fracture nonunions.

\section{Types of interventions}

Subjects were given daily or weekly subcutaneous TPTD.

\section{Outcome measures}

Radiographs were done serially to assess callus formation until complete bone healing was noted. Clinical union was also considered in some reports.

\section{METHODOLOGY}

\section{Search strategy}

Language

Both published and unpublished English language studies were sought. Assessment for inclusion of 
foreign language publications was based on the English language extract, and if considered appropriate, an English translation of the study was sought.

\section{Bibliographic databases and keywords}

The keywords used to search for studies included in this review are: teriparatide, PTH, fracture, non-union.

Databases were searched for all the years available since the introduction of TPTD. Studies identified during the database searches were assessed for relevance from a review of the title, abstract and descriptors of the study. A full text report was obtained for all the studies deemed relevant for this review. The search included the following databases:

Cochrane library, OVID, Google scholar, Science Direct, PubMed and Springerlink

\section{Hand searching}

The reference lists of all identified publications (both included and excluded) were searched for additional studies.

\section{SUMMARY OF CASE REPORTS}

\section{Femoral Neck Nonunion}

Y. Mitani reported an 88-year-old female with a femoral neck fracture who underwent surgery with a Hansson hook-pin system. Medical history included colon and gastric cancer. She also has rheumatoid arthritis with daily intake of $50 \mathrm{mg}$ bucillamine and $5 \mathrm{mg}$ prednisolone daily for more than 10 years. At 11 months, a diagnosis of non-union was made. TPTD $56.5 \mathrm{mcg}$ was administered on a weekly basis and complete union was obtained at 23 months. Serum examination after 36 weekly injections of TPTD was as follows: C-reactive protein, $0.2 \mathrm{mg} /$ $\mathrm{dL}$; rheumatoid factor, $170 \mathrm{U} / \mathrm{ml}$; alkaline phosphatase, $208 \mathrm{U} / \mathrm{l}$; albumin, $4.0 \mathrm{~g} / \mathrm{dL}$; calcium, 8.8 $\mathrm{mg} / \mathrm{dL} .[7]$

\section{Femoral Shaft Nonunion}

Y. K. Lee, et al., reported three cases lage range 29-68 years) who had non-union of the femur even after initial surgical intervention. TPTD was administered for three months after a diagnosis of non-union. All patients denied smoking and alcohol abuse, and had no history of metabolic disease or glucocorticoid use. They also had no clinical sign of infection and the laboratory tests indicative of infection or defective bone healing were normal. A successful union was obtained in all three patients without further surgical intervention and no adverse events related to the use of TPTD were observed. Their report showed that TPTD could be an alternative to surgical intervention in non-union of the femur.[6]

In another case by Wei, et al., they reported a patient who underwent intramedullary nailing and was subsequently exchanged with a plate with autogenous bone graft. They showed that TPTD could successfully treat a femoral fracture nonunion that autogenous bone grafting failed to heal after 1 year. Laboratory tests, including white blood cell counts, C-reactive protein and erythrocyte sedimentation rate were normal excluding infected non-union. The patient denied smoking and alcohol abuse and had no history of glucocorticoid use. Central dual-energy $x$-ray absorptiometry revealed normal bone mineral density. Other laboratory tests, including serum alkaline phosphatase, PTH, calcium, creatinine and $25(\mathrm{OH})$ vitamin $D$ were normal excluding any metabolic disorder. Successful union was obtained after once-daily administration of TPTD $(20 \mathrm{mcg} /$ day) for nine months.[17]

A report in Japan by K. Nozaka, et al., states that they combined the effect of TPTD and low-intensity pulsed ultrasound for union. A 56-year-old male with a femoral shaft fracture underwent intramedullary osteosynthesis nailing initially. He had no radiological or clinical sign of healing three months later and low-intensity pulsed ultrasound was initiated at that time. He was reassessed in another three months with evidence of mild bone consolidation but the fracture gap persisted. Subsequent treatment with human PTH was initiated in combination with low-intensity pulsed ultrasound. Full fracture healing was present six months after beginning the combination low-intensity pulsed ultrasound and TPTD. It is hypothesized that the potential additive effects of low-intensity pulsed ultrasound and TPTD therapy ultimately triggered sufficient bone formation to support osseous union.[7]

\section{Femoral and Tibial Nonunion}

In a report by L. Xiaofeng, et al. in China, a 44-yearold male suffered closed fractures of the right tibia 
and left femur and underwent intramedullary nailing. He had no postoperative infection. Medical history taking revealed no chronic diseases, hypertension (HTN), diabetes mellitus (DM), smoking, alcohol abuse, or regular medication. Until 10 months postoperatively, there was no radiographic healing of the right tibia, and the patient experienced persistent pain during weight-bearing. Radiographs obtained 11 months postoperatively revealed a persistent fracture gap, sclerosis of the fracture margin, and no evidence of bone bridging at the fracture site. There was no clinical sign of infection based on erythrocyte sedimentation rate, C-reactive protein level and leukocyte count. Other laboratory tests, including PTH, serum alkaline phosphatase, phosphorus, calcium and 1,25-(OH)2D3 were within normal range. TPTD was started at $20 \mathrm{mcg}$ daily and after four months radiographs showed a decrease of the fracture gap and bone showed complete fracture union.[16]

\section{Non-union of Open Type Fractures}

In 2015, C. Coppola, et al., reported four patients who received TPTD for the management of non-union after open fixation of traumatic fractures of the lower limb.

The first case is a 36-year-old male healthy swimmer, non-smoking, who sustained a Gustilo III 3B fracture of the right femur, 33A3.1. He underwent open reduction and monoaxial external fixation supplemented by Kirschner wires. After 4 weeks, an external fixator was applied. Radiographs after two months showed poor bone apposition in the medial and posterior aspects of the fracture site. Four months after the initial injury, the distal external fixation pins had loosened and there was a delay in healing. The patient underwent open reduction, fixation and stabilization of the fracture with a monoaxial fixator supplemented with Allomatrix (Wright Medical Technology, Inc, Arlington, Tenn). Eleven months after injury, despite signs of non-union at radiographic evaluation, the external fixator was removed and the patient started to walk in a protected brace. Laboratory investigations, including serum alkaline phosphatase, PTH, calcium, creatinine and $25(\mathrm{OH})$ vitamin $D$ were normal, excluding any metabolic disorder. Fifteen months after the injury, the patient underwent open reduction and internal fixation with a condylar plate, application of platelet rich plasma and implantation of bone allograft. At 20 months, given the poor bone integration of the graft and the absence of bone callus, the patient started treatment with subcutaneous injection of TPTD $(20 \mu \mathrm{g}$ daily), calcium and vitamin D. After nine months of treatment, the 3D CT showed complete integration around the plate and the bone graft with adequate formation of bone callus at the site of non-union. The serum levels of alkaline phosphatase increased during the nine months of therapy with TPTD normalized within three months of interruption of TPTD administration. At follow-up three and five years from the last operation, the non-union was healed and the patient was satisfied in terms of daily and sport activity.

The second patient is a 33-year-old healthy non-smoking male who underwent reduction and external fixation of a Gustilo III 3B fracture of the right tibia and fibula, 42A.2. Five months from surgery, given the absence of any signs of healing on radiographs, the patient underwent open reduction and reamed intramedullary nailing. Five months from the nailing, the patient continued to report pain and was only able to partially weight-bear with bilateral elbow crutches. Radiographs showed lack of evident callus and atrophic nonunion. The patient started treatment with TPTD $(20 \mu \mathrm{g}$ subcutaneous injection daily), calcium and vitamin D. After four months of TPTD administration, radiographs showed good integration of the bone around the nail, and adequate bone callus over the site of non-union. Ten months later, he returned to his preoperative occupation of insurance salesman and sport activities (modern pentathlon).

The third patient is a 28-year-old healthy male non-smoking truck driver who sustained a Gustilo III $3 B$ fracture of the left femoral shaft 32B.2 and left tibia and fibula $43 \mathrm{~A}$ after a road accident. He underwent reduction and internal fixation using a reamed retrograde femoral nail, and external fixation of the tibia-fibula fracture. At four months, radiographs showed poor healing, lack of bone callus and atrophic nonunion for both fractures.

The patient underwent removal of the external fixator of the lower leg and started treatment with TPTD (20 $\mu \mathrm{g}$ subcutaneous injection daily), calcium and vitamin D. After three months of therapy, radiographs showed good integration of the bone around the nail and adequate callus over the site of non-union. Twelve months later, he started to work again and had changed sport. Flexion of the knee was to $95^{\circ}$, extension was full. 
The fourth patient is a 30 -year-old healthy engineer, non-smoking male who underwent reduction and external fixation (Hoffmann II device) of 43A.3 Gustilo III 3B fracture of the left tibia and fibula. At four months, radiographs did not show any signs of bone healing. At that time, the patient underwent removal of the external fixator and administration of TPTD (20 $\mu \mathrm{g}$ subcutaneous injection daily), calcium and vitamin D. Given the soft tissue impairment, he could only undergo hyperbaric therapy and plastic surgical procedures. After four months of therapy, radiographs showed good integration of the bone and adequate bone callus over the site of non-union. Eight months later, he could work and swim. Ankle extension was $10^{\circ}$, flexion $20^{\circ}$.

\section{Radial Neck Nonunion}

B. Garg, et al. reported a 60-year-old female who underwent an open reduction and internal fixation with a $2.00 \mathrm{~mm}$ T-plate. However, the fracture failed to unite ever after one year. They investigated the patient for factors responsible for non-union. Her personal history was negative in terms of any other medical illness, smoking, alcohol intake or any other form of drug intake. Her serum biochemistry parameters, PTH and vitamin D level were within normal range. However, her DEXA scan revealed severe osteoporosis for which she was started on injection TPTD $20 \mathrm{mcg} s / \mathrm{c}$ daily. When she reported after three months for follow-up, her pain had resolved and radiographs revealed union of the radial neck fracture.[5]

\section{Humeral Nonunion}

E. Ciurlia, et al. reported a 38-year-old male with a diaphyseal comminuted humeral fracture. He underwent osteosynthesis with bridge plating. At four months it did not show signs of healing. The patient underwent revision surgery eight months after the first surgery with an anterograde intramedullary nailing and autologous iliac bone grafts. X-rays achieved three months after the revision surgery did not show any signs of healing. This was consistent with the clinical manifestations of pain, movements at the fracture site and left arm weakness. The physical examination and laboratory tests, including white blood cell counts, C-reactive protein and erythrocyte sedimentation rate were normal, which permitted to rule out underlying infection. The patient denied smoking, alcohol abuse and history of metabolic disease. Glucocorticoid intake $20 \mathrm{mcg} /$ day of TPTD was given for three months and radiographs showed fracture healing.[2]

In a report by A. Oteo-Alvaro, et al., a 39-yearold male, smoker of 10-15 cigarettes daily, diagnosed with paranoid schizophrenia and depressive syndrome was being treated with olanzapine $5 \mathrm{mg} /$ day, lormetazepam $1 \mathrm{mg} /$ day, clorazepate dipotassium $45 \mathrm{mg} /$ day in three doses and lorazepam 3 $\mathrm{mg} /$ day in three doses. He had cocaine and alcohol addiction problems and was voluntarily participating in a methadone treatment program. The patient sustained a multifragmented fracture of the right humerus. Normal results were reported for the following laboratory parameters among others: total alkaline phosphatase and its bone fraction, liver function tests, including negative serologic tests for hepatitis $B, C$ and HIV, serum creatinine, ionic calcium, erythrocyte sedimentation rate, $C$ reactive protein, 25-hydroxy-vitamin D, parathyroid hormone, prolactin, testosterone and 24-hour urinary calcium. Dual energy $x$-ray absorptiometry (DXA scan) revealed normal values (lumbar spine DXA scan $T$ score- 0 , 9; total hip T score-0, 8; femoral T score-0, 9). A non-operative treatment consisted of immobilization with plaster followed by a functional brace. At seven months, radiographs showed non-union. After four months of TPTD therapy, radiographs showed complete union.[10]

In a report by $A$. Oteo-Alvaro, a right-handed Caucasian 32-year-old white fireman had a ski accident in January 2009. He was diagnosed with right diaphyseal humeral fracture and underwent surgery at another hospital consisting of intramedullary osteosynthesis with Hackethal technique with two elastic nails after failed manipulation the day before. Radiologic controls at five and six months did not show any signs of healing. This was consistent with the clinical manifestations of pain, movement at the fracture site and right arm weakness. The physical examination and laboratory tests, including white blood cell counts, C-reactive protein and erythrocyte sedimentation rate were normal, which permitted to rule out underlying infection. The patient denied smoking, alcohol abuse, and had no history of metabolic disease or glucocorticoid intake. He was treat- 
ed with NSAIDs (ibuprofen $1800 \mathrm{mg} /$ day) up to the third week after surgery. Other laboratory data, including serum alkaline phosphatase, PTH and calcium were normal. The patient declined to be reoperated on and consented to an empiric, off-label therapy with TPTD at approved doses for the treatment of osteoporosis (20 mg/day) as an attempt to treat the atrophic nonunion. After three months of treatment with TPTD, an x-ray showed the presence of bone bridges and a decreased gap between fragments. After five months of treatment, healing was complete, coinciding with the disappearance of pain and a complete range of shoulder and elbow joint mobility. Treatment with TPTD was discontinued. No side effects attributable to the drug were observed during treatment; subsequent laboratory tests continued to be normal.[11]

\section{Nonunion After an Ankle Arthrodesis}

This is a case report of a 25 -year-old diabetic female by K. Tamai, et al. who sustained a femoral shaft fracture and a non-union after multiple ankle arthrodesis for Charcot arthropathy 15 months prior to her femoral shaft fracture. Her laboratory data, including serum levels of alkaline phosphate, PTH, calcium and phosphorus were normal, but her level of 1.25 vitamin D3 was low and her left femoral bone density was extremely low $(0.54 \mathrm{mg} / \mathrm{cm} 2)$ and 2.0 standard deviations below the normal value for her age. Femoral fixation was done with an intramedullary nail fixation and she was immediately started with $20 \mathrm{mcg}$ subcutaneous injection of TPTD. After 12 weeks, there was complete union of the ankle.[14]

\section{Sternal Nonunion}

S. Chintamaneni, et al. reported a 67-year-old male who sustained a fracture of the body of the sternum. Atrophic nonunion was demonstrated by computed tomography and magnetic resonance imaging of the chest. The patient's other medical problems included HTN, Raynaud's phenomenon and glaucoma. He had no known metabolic disease. Laboratory studies revealed normal calcium, $25(\mathrm{OH})$ vitamin $\mathrm{D}$, total alkaline phosphatase and intact PTH. The remainder of the comprehensive metabolic panel, complete blood count, sedimentation rate and thyroid function tests were also normal. Central dual-energy x-ray absorptiometry revealed normal bone mineral density. Daily TPTD $20 \mathrm{mcg}$ subcutaneous injections were initiated and there was complete healing after nine months.[3]

\section{Periprosthetic Nonunion}

A report on a non-union on a periprosthetic fracture in a total knee arthroplasty (TKA) was reported in Japan by K. Ochi, et al. The patient is a 74-yearold female with a 26-year history of rheumatoid arthritis (RA) who sustained a periprosthetic femoral fracture owing to a fall from the stairs that occurred 13 years after she had undergone TKA. The fracture was internally fixed with a periarticular locking plate and hydroxyapatite graft and additionally treated with daily low-intensity pulsed ultrasound. However, non-union of the fracture became apparent when the locking plate broke nine months after the internal fixation. Thus, the fracture was consequently treated with a combination of an additional internal fixation with a locking plate and bone grafts (autograft and allograft) and daily low-intensity pulsed ultrasound. However, although radiographs of the distal femur showed some external callus formation, a computed tomography (CT) scan revealed signs of non-union la gap remaining between the fracture fragments with sclerotic margins) nine months after the consequent internal fixation (Figure 1a). Thus, the patient was additionally treated with once-weekly TPTD injections nine months after the consequent internal fixation. After two months of treatment with TPTD, the CT images showed a decreased gap between the two fragments and showed new bone filling between the fracture gap after six months (24 months after the primary internal fixation and 15 months after the consequent internal fixation), suggesting fusion of the non-union. [9]

In another report by $C$. Emanuele in Italy, it reported a case of a 64-year-old female with a non-union of a periprosthetic fracture at the distal end of the humeral stem of RSA (reverse shoulder arthroplasty) positioned three years before. Immobilization with an arm brace was used as the first conservative treatment. After failed conservative treatment, the patient underwent plate osteosynthesis with plate, screws and wires in addition to a massive bone autograft. Postoperatively, the patient followed a therapy with TPTD (20 mcg/daily for three months). X-ray control 


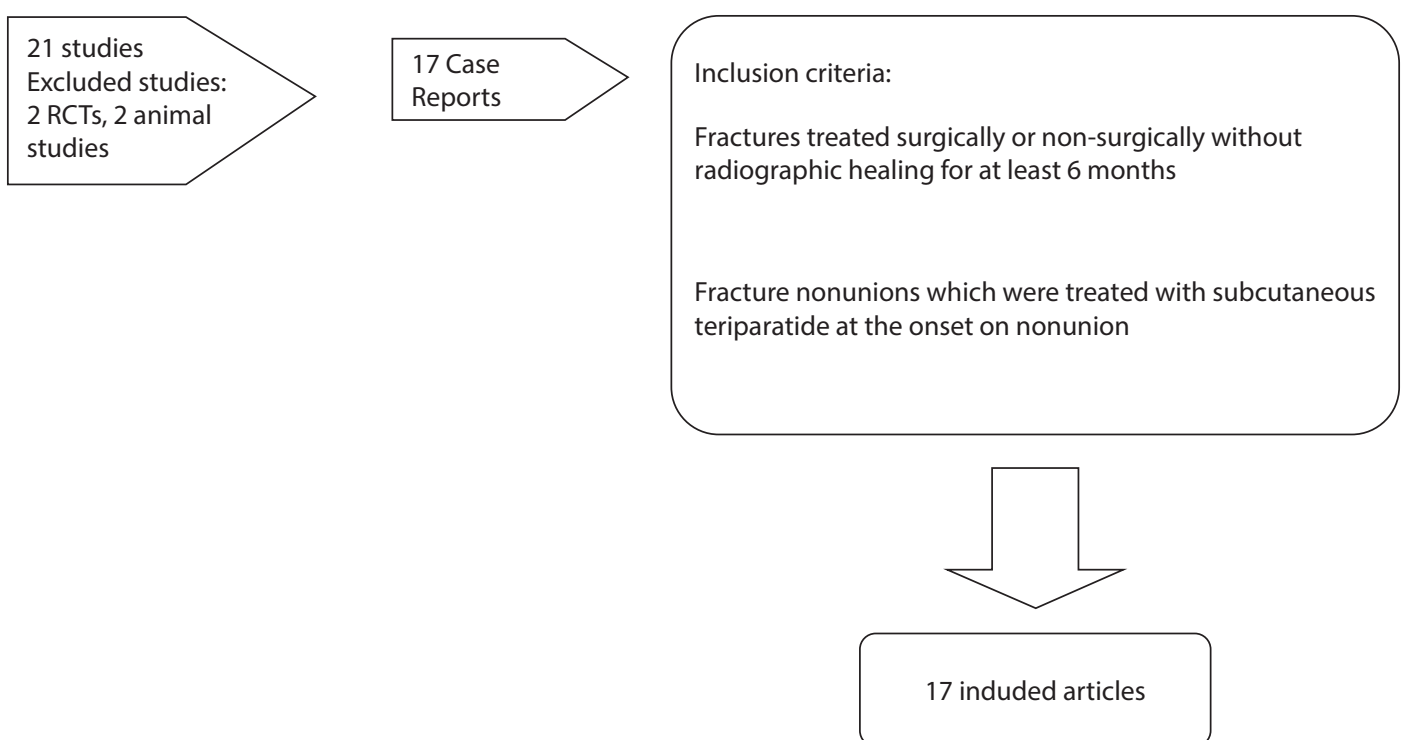

Figure 1. The results of the search strategy using key terms

was performed at three months. X-ray at one year postoperatively showed complete fracture healing. Clinical control was good (DASH score 76).[4]

\section{Nonunion After Ulnar Shortening Osteotomy for Smokers}

Uemura, et al. presented two cases of smokers, a 62-year-old male and a 42-year-old female, with non-union after ulnar shortening osteotomy and fixation with a 6-hole non-locking plate for ulnar impaction syndrome. For the treatment of non-union, noninvasive therapy with TPTD (20- $\mu$ g, subcutaneous injection) in addition to low-intensity pulsed ultrasound was initiated. In both cases, partial bone union began to be observed on radiographs after the first four weeks of TPTD administration and successful bone healing without additional surgical interventions was achieved after 10 and 6 months of treatment with TPTD, respectively.[15]

\section{Type II Dens Fracture Nonunion}

As reported by E. Pola, et al. last January 2017, they reported a 73-year-old female who sustained a type II odontoid fracture with anterior displacement. The patient elected for a non-surgical treatment. A Philadelphia collar was used to stabilize the fracture and the patient was discharged after three days. Six months following the injury, the patient was still complaining of significant axial neck pain. A computed tomography (CT) scan at six months after injury revealed non-union at the fracture site, distance between fracture fragments was $4 \mathrm{~mm}$ and there was evidence of sclerotic bone margins at the level of the fracture. The patient was maintained in a rigid collar and was offered off-label therapy with daily subcutaneous injections of TPTD (rhPTH1-34) 20 $\mu \mathrm{g} / \mathrm{d}$, which she accepted. This is the same regime used for treatment of osteoporosis in post-menopausal women. The anabolic treatment with TPTD was monitored through periodic examinations and regular measurements of serum levels of calcium, phosphorus, vitamin D, PTH and alkaline phosphatase. Forty-five days after starting TPTD treatment, an interval CT scan showed an initial phase of callus formation at the fracture site with partial closure of the fracture gap. Anabolic therapy was continued for three months. At the end of treatment a final CT scan confirmed a complete consolidation of the fracture. Flexion/extension x-rays of the cervical spine showed no residual instability and axial neck pain had resolved as well.[13]

\section{RESULTS}

A total of 22 reported cases in 17 articles were identified; those patients were reported from 2009-2017 and are summarized in Table 1 . The median reported age was 53 years (age range: 25-88 years). There were four patients with comorbidities (cancer, RA, DM, HTN). The median reported duration of treatment was 5.6 months (range: 3-10 months). In all, 22 patients were treated for non-union. The median documented time to complete union was 8.5 months (range: 3-23 months). There were three patients who 
had smoking histories. There were no reported adverse effects.

\section{DISCUSSION}

As shown by the case reports, union was achieved as early as three months in some cases but may take up to 23 months in young or old patients. Daily or weekly administration showed to have positive results. The reports also noted that TPTD was used even in the presence of comorbidities and patients with history of smoking. All reports showed no negative side effects during and after treatment. However, these are only few reported cases and their results are not conclusive and final.

The management of non-union is very challenging. Advancing age, diabetes, steroid use, osteoporosis, both mechanical and anatomical factors lead to impaired fracture healing. Undiagnosed metabolic or endocrine disorders of calcium, vitamin D and PTH are considered predisposing factors in non-union. Autologous bone grafting is the current gold standard to aid healing in atrophic non-unions, but postoperative pain, infection, nerve or vascular inju- ries, and donor site discomfort may complicate this procedure and this too may ultimately fail as shown in some of the cases mentioned above.

TPTD is of interest in the treatment of impaired fracture healing because it may stimulate new bone formation, increase early bone-implant contact, enhance the tensile strength of the bone-cement interface, increase the rate of successful union at the fracture site, and improve bone mineral content and callus volume and mineralization. Several clinical studies have shown that it accelerates fracture healing as shown in studies by Bhandari 2015, Aspenberg 2010 and Huang 2016. However, TPTD is still not approved or indicated for use in fracture healing.

\section{CONCLUSION}

This review suggests that there is indeed a role of TPTD in non-union, however, well designed RCTs are required to comprehensively analyze the actions of TPTD in human subjects. This will allow conclusive decisions to be made on whether or not to incorporate TPTD as a standard option for management of fracture non-unions. 


\section{REFERENCES}

1. Bhalla AK. Use of teriparatide (TP) in fracture healing. Bone. 2010(47):S27.

2. Ciurlia E, Leali PT, Doria C. Use of teriparatide off-label: our experience and review of literature. Clinical Cases in Mineral and Bone Metabolism. 2017 Jan; 14(1):28.

3. Chintamaneni S, Finzel K, Gruber BL. Successful treatment of sternal fracture nonunion with teriparatide. Osteoporosis International. 2010 Jun 1;21(6):1059-63.

4. Emanuele C, Leonardo P, Gianfilippo C, Matteo A, Doria C. Peri-prosthetic humeral non-union: Where biology meets bio-mechanic. A case report. International Journal of Surgery Case Reports. 2017 Jan 1;39:102-5.

5. Garg B, Batra S, Dixit V. An unexpected healing of an established non-union of the radial neck through teriparatide: A case report and review of literature. Journal of Clinical Orthopaedics and Trauma. 2018 Mar;9(Suppl 1):S103.

6. Lee YK, Ha YC, Koo KH. Teriparatide, a nonsurgical solution for femoral nonunion? A report of three cases. Osteoporosis International. 2012 Dec 1;23(12):2897-900.

7. Mitani Y. Effective treatment of a steroid-induced femoral neck fracture nonunion with a once-weekly administration of teriparatide in a rheumatoid patient: a case report. Archives of Osteoporosis. 2013 Dec 1;8(1-2):131.

8. Nozaka K, Shimada Y, Miyakoshi N, Yamada S, Hongo $M$, Kasukawa $Y$, et al. Combined effect of teriparatide and low-intensity pulsed ultrasound for nonunion: a case report. BMC Research Notes. 2014 Dec;7(1):317.

9. Ochi K, Ikari K, Naomi A, Momohara S. Administration of teriparatide treatment for a challenging case of nonunion of periprosthetic fracture after total knee arthroplasty. Archives of Osteoporosis. 2013 Dec 1;8(1-2):159.

10. Oteo-Álvaro Á, Marín MT. Nonunion of the humeral shaft successfully treated with teriparatide [rh (1-34) PTH]. Case Reports in Clinical Medicine. 2013 Mar 7;2(1):11-5.

11. Oteo-Álvaro Á, Moreno E. Atrophic humeral shaft nonunion treated with teriparatide (rh PTH 1-34): a case report. Journal of Shoulder and Elbow Surgery. 2010 Oct $1 ; 19(7): \mathrm{e} 22-8$
12. Pietrogrande L, Raimondo E. Teriparatide in the treatment of non-unions: scientific and clinical evidences. Injury. 2013 Jan 1;44:S54-7.

13. Pola E, Pambianco V, Colangelo D, Formica VM, Autore G, Nasto LA. Teriparatide anabolic therapy as potential treatment of type II dens non-union fractures. World Journal of Orthopedics. 2017 Jan 18;8(1):82.

14. Tamai K, Takamatsu K, Kazuki K. Successful treatment of nonunion with teriparatide after failed ankle arthrodesis for Charcot arthropathy. Osteoporosis International. 2013 Oct $1 ; 24(10): 2729-32$.

15. Uemura T, Okada M, Yokoi T, Shintani K, Nakamura H. Successful bone healing of nonunion after ulnar shortening osteotomy for smokers treated with teriparatide. Orthopedics. 2015 Aug 20;38(8):e733-7.

16. Xiaofeng L, Daxia X, Yunzhen C. Teriparatide as a nonoperative treatment for tibial and femoral fracture nonunion: $A$ case report. Medicine. 2017 Apr;96(16):e6571.

17. Yu W, Guo X. Teriparatide treatment of femoral fracture nonunion that autogenous bone grafting failed to heal: a case report. Archives of Osteoporosis. $2017 \mathrm{Dec} 1 ; 12(1): 15$.

18. Coppola C, Del Buono A, Maffulli N. Teriparatide in fracture non-unions. Translational Medicine@ UniSa. 2015 May; 12:47.

(c) (i) Open Access This article is licensed under a Creative Commons Attribution 4.0 International License, which permits use, sharing, adaptation, distribution and reproduction in any medium or format, as long as you give appropriate credit to the original author(s) and the source, provide a link to the Creative Commons license, and indicate if changes were made. The images or other third party material in this article are included in the article's Creative Commons license, unless indicated otherwise in a credit line to the material. If material is not included in the article's Creative Commons license and your intended use is not permitted by statutory regulation or exceeds the permitted use, you will need to obtain permission directly from the copyright holder. To view a copy of this license, visit http://creativecommons.org/licenses/by/4.0/. 